\title{
A Case Study in Automated Molecular Microscopy
}

Scott Stagg, James Pulokas, Denis Fellmann, Anchi Cheng, Joel Quispe, Satya Mallick, Radomir Avila, Clinton S. Potter and Bridget Carragher

Dept. of Cell Biology, National Resource for Automated Molecular Microscopy, The Scripps Research Institute, La Jolla, CA 92037

Over the past several years we have been developing an automated system, called Leginon, to acquire images from vitreous ice specimens $[1,2]$. One of the goals in developing an automated system for EM data acquisition system was to improve both the ease of use and the throughput of the process of acquiring low dose images of macromolecular specimens embedded in vitreous ice.

Using GroEL as a test specimen we have demonstrated the potential of the Leginon system for high-throughput data acquisition by acquiring images of more than 280,000 particles of GroEL in a single continuous session at the microscope. The only significant human intervention required was the periodic replenishment of liquid nitrogen in the cryostage and cold trap of the microscope. We have also demonstrated the potential for an automated pipeline for molecular microscopy by showing that these particles can be subjected to completely automated procedures to reconstruct a three dimensional (3D) reconstructed density map to a resolution better than $8 \AA$ (see Figure 1). These automated procedures include particle selection and CTF determination, which are two of the most arduous and tedious steps required for single particle processing. Visual examination of the output of the automated particle selection (using the Selexon software [3], see figure 2)) and the automated CTF determination (using the ACE software [4], see figure 3) confirmed the satisfactory performance of these packages. Integration of the Selexon and ACE software packages with the Leginon database greatly simplified the task of managing and manipulating the data and files. Together the Leginon, Selexon, and ACE packages provide a prototype for an automated pipeline where data are stored and managed using a relational database and a variety of web-based tools are used to examine and assess the outputs.

In generating the 3D maps we used a variety of metadata associated with the data acquisition and processing steps to sort and select the particles. These metadata provided a number of insights into factors that affect the quality of the acquired images and the resulting reconstructions. In particular we have shown that the resolution of the reconstructed three dimensional density maps improves with decreasing ice thickness. These data provide a basis for assessing the limitations and bottlenecks of high throughput macromolecular microscopy.

References

1. Carragher et al. (2000) J. Struct. Biol., 132, 33-45.

2. Suloway et al. (2005) J. Struct. Biol., 151, 41-60.

3. Zhu et al. (2001) J. Struct. Biol., 135, 302-312.

4. Mallick et al. (2005) Ultramicroscopy, 104, 8-29.

5. Ludtke, et al. (1999) J Struct Biol. 128, 82-97.

This research is supported by the National Institutes of Health though the National Center for Research Resources' P41 program (RR17573). 


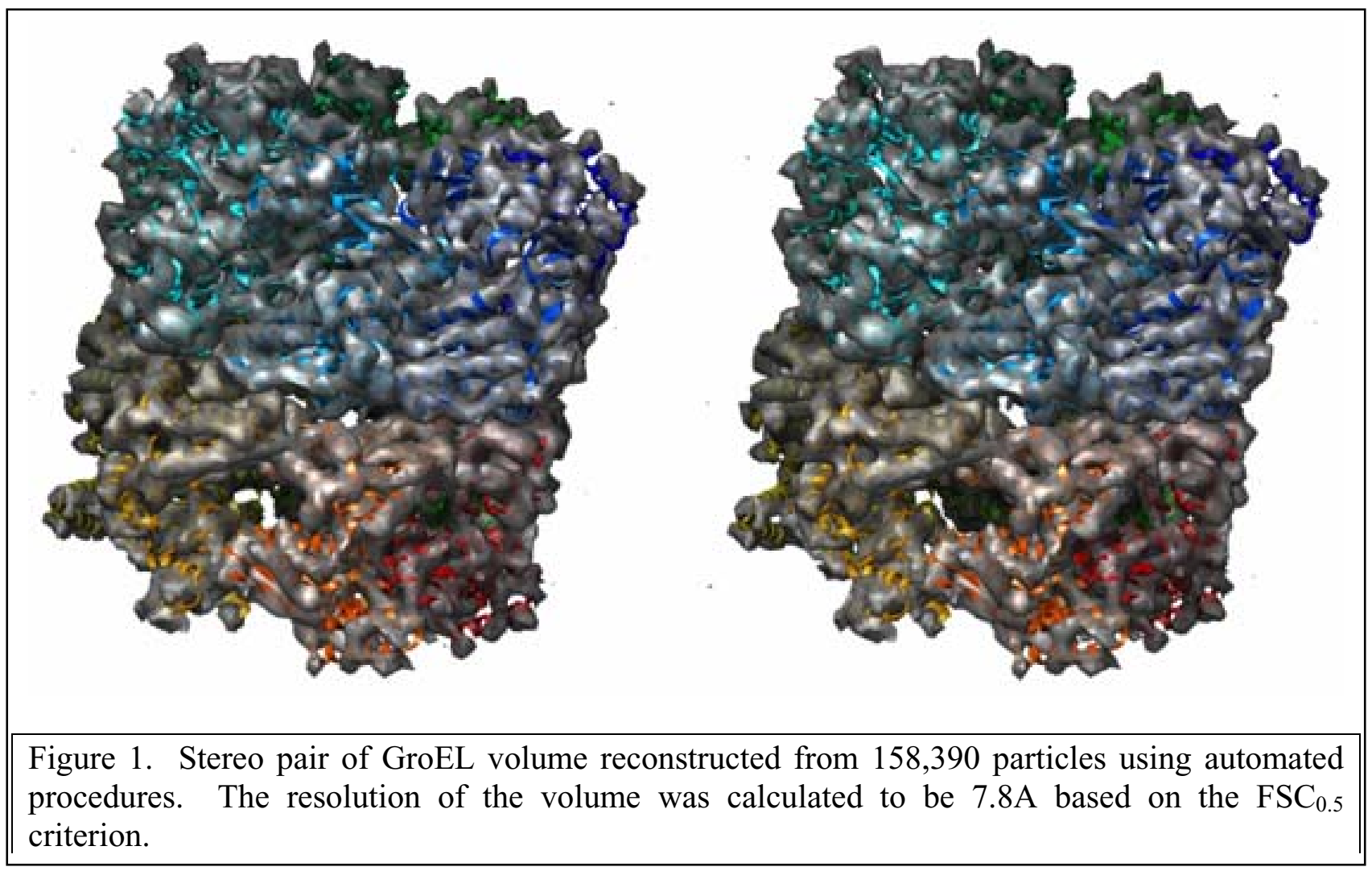

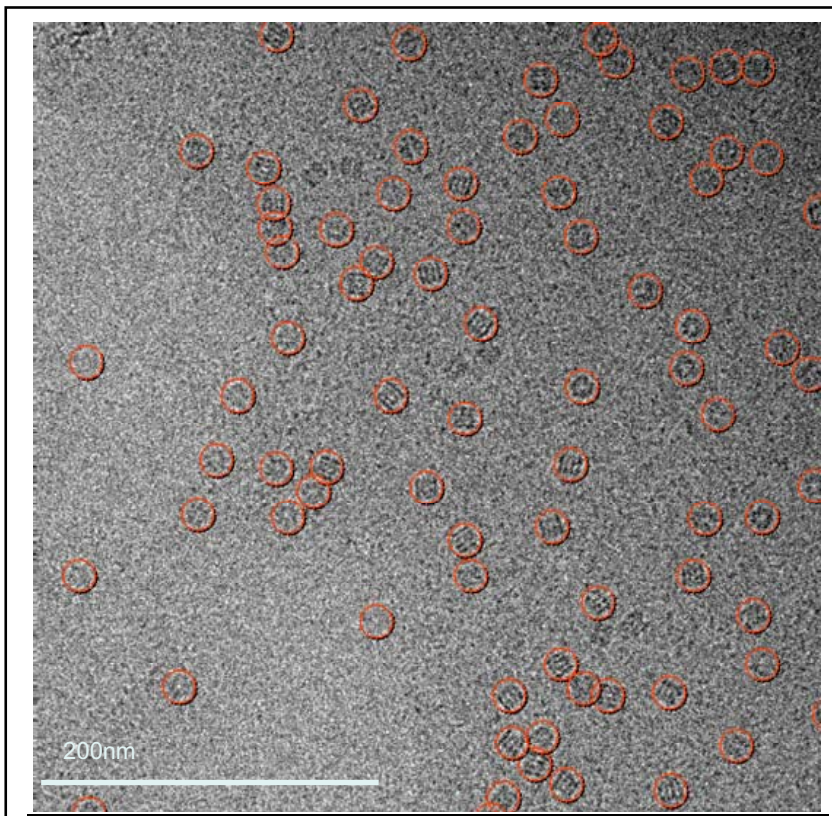

Figure 2. A small region of one of the 552 micrographs (nominal magnification 50,000x) acquired by Leginon. The individual GroEL particles automatically identified using Selexon are enclosed in the orange circles.

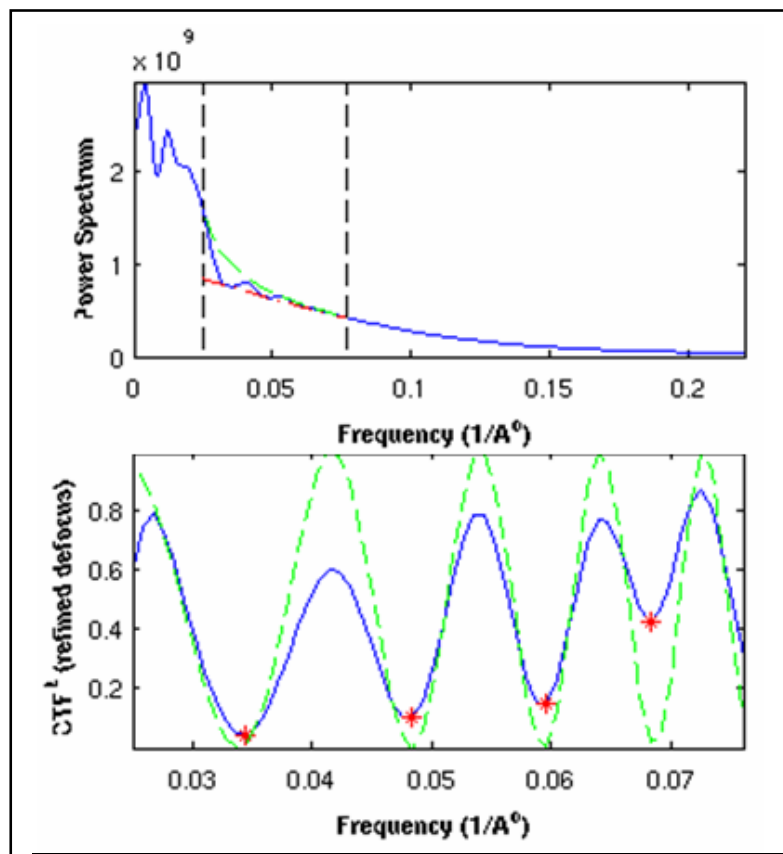

Figure 3. ACE provides an automated estimate of the CTF of an image (bottom) based on a rotationally averaged power spectrum (top). 